\title{
Is delayed graft function associated with ureteral stenosis in the kidney transplant recipient? A case-control study
}

Axel Cayetano-Alcaraz ${ }^{1}$; Juan Sebastian Rodriguez-Alvarez ${ }^{1}$; Mario Vilatobá-Chapa ${ }^{2}$; Josefina Alberú-Gómez ${ }^{2}$; Bernardo Gabilondo-Pliego ${ }^{1}$, Francisco Rodríguez-Covarrubias ${ }^{1}$; Luis Eduardo Morales-Buenrostro ${ }^{3}$; Carlos Enrique Méndez-Probst ${ }^{1}$

${ }^{1}$ Department of Urology, Instituto Nacional de Ciencias Médicas y Nutrición Salvador Zubirán, Mexico City, Mexico; ${ }^{2}$ Department of Transplants, Instituto Nacional de Ciencias Médicas y Nutrición Salvador Zubirán, Mexico City, Mexico; ${ }^{3}$ Department of Nephrology, Instituto Nacional de Ciencias Médicas y Nutrición Salvador Zubirán, Mexico City, Mexico

Cite as: Can Urol Assoc J 2019 February 26; Epub ahead of print. http://dx.doi.org/10.5489/cuaj.5794

Published online February 26, 2019

$* * *$

\section{Abstract}

Introduction: Ureteral stricture (US) in the kidney transplant recipient is a rare complication that can lead to morbidity and graft loss. Risk factor recognition is crucial in the prevention and management of this entity. Delayed graft function (DGF), as defined by the need for dialysis in the first week after transplantation, has been proposed as a risk factor in previous studies. Our objective is to determine the impact of DGF in US development in kidney transplant patients.

Methods: We designed a matched case-control study. US cases in kidney transplant recipients were identified in the 2008-2017 period. We defined US as the rise in serum creatinine associated with findings suggesting obstruction in ultrasound, scintigraphy, or retrograde pyelogram; any other cause of graft dysfunction was excluded. Controls were defined as kidney transplant recipients from the same population and period without US, matched in a 1:2 fashion by age, sex, and donor type.

Results: From 532 kidney transplant patients, 31 cases and 62 controls were included. Cumulative US incidence was 58 per 1000 cases. When calculating for odds ratio (OR), post-operative urinoma (OR 3.2; 95\% confidence interval [CI] 2.36-4.37) and ureteral duplication (OR 3.29; 95\% CI 2.40-4.51) were associated with an increased risk for US, while DGF was not found to be statistically significant as a risk factor (OR 3.3; 95\% CI 0.96-11.52). No statistically significant differences were found between groups in other pre- and post-transplant-related factors 
Conclusions: DGF was not associated with US in our cohort; however, ureteral duplication and postoperative urinoma were associated with an increased risk of graft ureteral stenosis development.

\section{Introduction}

Kidney transplantation is the definitive treatment for chronic kidney failure, bearing an improvement in prognosis over dialysis. ${ }^{1,2}$ Urologic complications are highly relevant as they may end up in graft loss. ${ }^{3,4}$ In particular, ureteral complications have been reported from $4.8 \%$ to $9.2 \%$, with ureteral stenosis (US) rates from $2.4 \%$ to $9.2 \%$ of the kidney transplants. ${ }^{5-7}$ Additionally, most ureteral complications occur during the first posttransplant year. ${ }^{5,8,9}$

Another feared complication is delayed graft function (DGF), which is associated with an increased risk for graft loss and acute rejection in the first post-transplant year. ${ }^{10}$ This complication has been reported from $2 \%$ to $50 \%$ and $1.6 \%$ to $10 \%$ in deceased and living donor transplants, respectively. ${ }^{11,12}$ The primary underlying etiology for DGF seems to be ischemia-reperfusion damage. ${ }^{11}$

Some retrospective studies have set a role for DGF as a risk factor for US in renal grafts, along with other variables such as donor age over 65 years and kidneys with more than two arteries. ${ }^{5}$

We hypothesize that DGF increases the risk for graft US secondary to ischemiareperfusion damage, pro-fibrotic molecule expression and ureteral ischemia that promote aberrant scarring. ${ }^{13,14}$

Our primary objective is to determine the impact of DGF in US development in kidney transplant patients that underwent transplantation in the 2008 to 2017 period in a tertiary care hospital.

Secondarily, to calculate the graft US prevalence, describe other risk factors and treatment modalities used for the resolution of this complication.

\section{Methods}

We performed a matched case-control study in a single center in Mexico from January 2008 to January 2017. We obtained approval from the local ethics board committee. Data were retrieved from the hospital kidney transplant database. Cases were kidney transplant receptors diagnosed with graft US, and controls were patients from the same population that did not develop US during follow-up.

\section{Case inclusion and exclusion criteria}

Cases were included as patients older than 18 years with a kidney transplant performed in this institution coupled with a confirmed diagnosis of graft US.

We defined graft US as a rise in serum creatinine associated with hydronephrosis on ultrasound, an obstructive curve in scintigraphy or a retrograde pyelography compatible 
with ureteral stenosis that were managed surgically (ureteroneocystostomy, Boari flap ureteroneocystostomy or ureteroureterostomy), endoscopically (retrograde double J stent catheterization, balloon dilation or ureterotomy) or by interventional radiology treatment (nephrostomy or antegrade double J stent catheterization). Any other potential cause such as rejection (acute or chronic) or obstructive uropathy of other etiology were excluded. Patients that did not undergo kidney transplantation in our institution were excluded. We eliminated patients with incomplete follow-up or a follow-up period of fewer than 3 months. Cases were matched with controls in a 1:2 fashion by age, sex, donor type and transplantation period $( \pm 5$ years).

\section{Control inclusion and exclusion criteria}

Controls were selected as kidney transplant recipients from either living or deceased donor older than 18 years that underwent transplantation in this institution during the same follow-up period of the cases with no graft dysfunction unless concluded to be a DGF. We excluded patients that did not undergo transplantation in our institution. Elimination criteria were an incomplete follow-up or a follow-up period of fewer than three months.

\section{Clinical variables}

- Donor: Age, sex, blood type, donor type (living or deceased), extended criteria, donor relation (related or unrelated), comorbidities and glomerular filtration rate (calculated with the MDRD formula).

- Receptor: Age, sex, blood type, comorbidities, body mass index, smoking status, previous transplantation, HLA mismatch, pre-transplant anti-T panel reactive antibodies (PRA \%), donor-specific HLA antibodies, pre-transplant diuresis, induction and maintenance scheme, BK virus infection, CMV infection, 5-day follow-up ultrasonography data (hydronephrosis, perigraft fluid collection, distal intrarenal resistive index), perioperative complications (lymphocele, hematoma, urinoma, urinary fistula, arterial anastomosis stenosis), positive urine culture, acute rejection.

- Renal graft: ischemia time, $\geq 2$ graft arteries, $\geq 2$ graft veins, ureteral duplication, ureteral implant technique, ureteral stent use, surgical bleeding, surgical time, time to stent withdrawal, ureteral stenosis diagnostic method, time to ureteral stenosis development, treatment type (surgical, endoscopic, interventional).

- Delayed graft function: Defined as the need for dialysis in the first post-transplant week. ${ }^{11,15,16}$ 


\section{Sample size}

We calculated a sample size of 216 patients, 72 cases and 144 controls. The figures were calculated with a power of $80 \%$, 95\% confidence interval, a 2 control per case proportion, with a $9 \%$ estimated control exposure and a minimal odds ratio detection of 3. (Fleiss, Statistical Methods for Rates and Proportions, formulas 3.18 \&3.19, results obtained from Dean AG, Sullivan KM, Soe MM. OpenEpi: Open Source Epidemiologic Statistics for Public Health. Available at www.OpenEpi.com, updated 2013/04/06, accessed 2017/04/10).

\section{Statistical analysis}

Data were analyzed with central tendency measures. Quantitative and qualitative data were compared using the student t-test and Chi-square, respectively. A p-value under 0.05 was considered statistically significant. We obtained odds ratio (OR) for significant differences with the Mantel-Haenzel test. Statistical Package for the Social Sciences (SPSS ${ }^{\circledR}$ ) version 20 was used for calculations.

\section{Results}

We reviewed 532 kidney transplantations in the 2008 - 2017 period, 31 cases and 62 controls were included. Median follow-up was 60.09 months (IQR 46.7). Table 1 describes the basal recipient and donor pre-transplantation characteristics; table 2 describes perioperative transplant variables and table 3 describes follow-up kidney receptor characteristics and immunosuppressive schemes.

A ureteral stent was placed in 28 (90.3\%) cases, and 54 (87.1\%) controls without significant statistical differences $(\mathrm{p}=0.206)$; median time for ureteral stent withdrawal was 29.68 days (SD 15.9) and 34.02 days (SD 20.3) for cases and controls, respectively, also with no statistical significance $(\mathrm{p}=0.3)$. Immunosuppressive regimes for induction and maintenance did not show statistical significance among groups.

\section{Odds ratio (OR) calculations}

In the risk estimation tests, Urinoma and ureteral duplication were the only variables associated with an increased risk of ureteral stenosis (Table 4).

\section{Characteristics of cases with graft ureteral stenosis}

The calculated incidence of US was 5.8\% while the incidence rate was 0.093 person-years. The median time for US development was 62 days (IQR 64). Imaging studies for US diagnosis were distributed as 28 (90.3\%), 7 (7.5\%) and 13 (41.9\%) cases for an ultrasound, renal scintigraphy, and retrograde pyelogram, respectively. US management was surgical in 28 cases (90.3\%), balloon dilation and retrograde stenting in 2 cases (6.4\%) and one case required interventional treatment with antegrade stenting. Surgical management was of 23 (74.2\%) with ureteral neocystostomy and 5 (15.1\%) with the Boari flap technique. 


\section{Discussion}

In our sample of 532 kidney transplants performed in the research period, the incidence of US was of 5.8\% with an incidence rate of 0.093 person-years, slightly higher than international series: for instance, in a series of 2000 transplants performed in a single medical center from 1980 through 2010, the documented US prevalence was $2.7 \% .{ }^{6}$ A rate of 3.5\% in an 894 transplant sample from 1093 through 2009 was reported by Faenza et al. ${ }^{5}$ Karam et al. reported a 4.1\% rate in 1787 transplants from 1990 through 2002. ${ }^{8}$ More recently, US was reported in $3.39 \%$ from a 973 transplant sample performed in the 2004 to 2014 period, with a mean time to stenosis of $10.6 \pm 23.0$ months (range: 0.5 - 98 months). ${ }^{4}$ Time to stenosis occurrence is related to its etiology. Early stenosis can be associated to tissue edema, kink of the ureter, narrow ureter diameter or extrinsic compression by hematomas or lymphoceles; ${ }^{17,18}$ while the late US can be associated to inappropriate arterial blood supply that results in ischemia and fibrosis. ${ }^{3}$ Nevertheless, definitions vary among studies, are not based on international consensus and tend to be arbitrary. ${ }^{3,5,6,8}$ In the present study, early US (defined as less than 3 months post-transplant) was more common, with a proportion of $74.2 \%(\mathrm{n}=23)$ and mean time from transplantation to US diagnosis was 62 days (IQR 64 days). Late US occurred in $25.8 \%(n=8)$ of cases. This proportion is similar to the one reported by Faenza et al. (81.2\% early stenosis vs. $18.8 \%$ late stenosis). ${ }^{5}$

DGF is a relatively common early post-transplant complication with a rate of up to $50 \%$ of deceased donor transplantations. ${ }^{19}$ Conversely, its occurrence in living donor transplantations is fairly uncommon, with rates reported from $1.6 \%$ to $10 \%(11,12)$. DGF has been associated with graft dysfunction, rejection in the first year and decreased graft survival. ${ }^{20}$ Some studies have proposed DGF as a risk factor for US: in 2006, Karam et al. in a single center retrospective analysis of 1787 patients with renal transplantation found that US was correlated to DGF $(\mathrm{p}=0.016)$, donor age $>65$ years $(\mathrm{p}=0.001)$ and more than two graft arteries ( $\mathrm{p}=0.009$ ); however, multivariate analysis showed only DGF (OR 1.03, CI95\% 1.01-1.05) and $>2$ arteries (OR 1.45, CI95\% 1.00-2-00) as an independent risk factor for US. ${ }^{8}$ Fontana et al. found similar results. ${ }^{7}$ Nonetheless, DGF by previous groups was defined as the number of days to reach a GFR of $>10 \mathrm{~mL} / \mathrm{min}$, and the study methods did not control potential confounding factors such as donor age and type.

At the moment, there is no consensus about a superior definition for DGF but the most commonly used is the need of dialysis in the first post-transplant week: it has been suggested to be adopted as the universal clinical variable for research studies. ${ }^{15}$ In our study of matched case-control analysis, although DGF was found to have a statistically significant difference in frequency between cases and controls by Chi-square analysis, we rejected the hypothesis of DGF as a risk factor for US considering the Mantel-Haenzel analysis did not show a statistically significant effect of exposure (OR 3.3, CI95\% 0.9611.52). Despite being associated with US in previous studies, our contrasting results may owe to different study design and previous non-standardized definitions for DGF. ${ }^{7,8}$ 
However, the associated factor may not DGF per se but one of the associated mechanisms such as ischemia-reperfusion damage, which may not always reach a clinical threshold to be considered as such.

The factors that we found for an increased risk of US were perioperative urinoma (OR 3.2, CI95\% 2.36-4.37) and ureteral duplication (UD) (OR 3.29 CI95\% 2.40-4.51). Previous studies did not report perioperative complications as risk factors for US. ${ }^{7,8}$ However, urinoma is commonly associated with a ureterovesical (UV) anastomotic leak or distal ureteral ischemia depending on the occurrence time, leading to periureteral graft fibrosis. ${ }^{21,22}$

On the other hand, UD is a common anatomy variant reported in 1 of 100-500 cases; mixed results have been reported in small studies where it may have been associated to an increased incidence of urologic complications (10.5\%), mainly US, ${ }^{23}$ while other studies show no difference in perioperative complications. ${ }^{24} \mathrm{~A}$ higher technical difficulty performing the UV anastomosis may explain the association between ureteral duplication and US.

Since US is regarded as an uncommon complication in renal transplantation, the study design appropriately evaluated the associated risk factors with this entity. Furthermore, the research study period is recent, and distinct renal transplant management aspects (such as patient selection, protocol follow-up, immunosuppressive schemes, and surgical groups) were standardized since the beginning of the period. Our study limitations are mainly a failure to achieve the optimal sample size owing to the rarity of this entity, as well as its single center and retrospective nature.

\section{Conclusion}

Delayed graft function was not associated with ureteral stenosis in our cohort; however, ureteral duplication and post-operative urinoma were associated with a graft ureteral stenosis development. 


\section{References}

1. Wolfe RA, Ashby VB, Milford EL, Ojo AO et al. Comparison of mortality in all patients on dialysis, patients on dialysis awaiting transplantation, and recipients of a first cadaveric transplant. $N$ Engl J Med 1999;341(23):1725-30.

2. Schold JD, Buccini LD, Goldfarb DA, et al. Association between kidney transplant center performance and the survival benefit of transplantation versus dialysis. Clin J Am Soc Nephrol 2014;9(10):1773-80.

3. Palazzetti A, Oderda M, Dalmasso E, et al. Urological consequences following renal transplantation: a review of the literature. Urologia 2015;82(4):211-8.

4. Gil-Sousa D, Oliveira-Reis D, et al. Ureteral Stenosis After Renal TransplantationA Single-Center 10-Year Experience. Transplant Proc 2017;49(4):777-82.

5. Faenza A, Nardo B, Fuga G, et al. Urological complications in kidney transplantation: ureteroneocystostomy versus uretero-ureterostomy. Transplant Proc 2005;37(6):2518-20.

6. Eufrasio P, Parada B, Moreira P, et al. Surgical complications in 2000 renal transplants. Transplant Proc 2011;43(1):142-4.

7. Fontana I, Bertocchi M, Rossi AM, et al. Late ureteral stenosis after kidney transplantation: a single-center experience. Transplant Proc 2010;42(4):1174-5.

8. Karam G, Hetet JF, Maillet F, et al. Late ureteral stenosis following renal transplantation: risk factors and impact on patient and graft survival. Am J Transplant 2006;6(2):352-6.

9. Rajpoot DK, Gomez A, Tsang W, et al. Ureteric and urethral stenosis: a complication of BK virus infection in a pediatric renal transplant patient. Pediatr Transplant 2007;11(4):433-5.

10. Yarlagadda SG, Coca SG, Formica RN, et al. Association between delayed graft function and allograft and patient survival: a systematic review and meta-analysis. Nephrol Dial Transplant 2009;24(3):1039-47.

11. Perico N, Cattaneo D, Sayegh MH, et al. Delayed graft function in kidney transplantation. Lancet 2004;364(9447):1814-27.

12. Park HS, Hong YA, Kim HG, et al. Delayed graft function in living-donor renal transplantation: 10-year experience. Transplant Proc 2012;44(1):43-6.

13. Ponticelli C. Ischaemia-reperfusion injury: a major protagonist in kidney transplantation. Nephrol Dial Transplant 2014;29(6):1134-40.

14. Eltzschig HK, Eckle T. Ischemia and reperfusion--from mechanism to translation. Nat Med 2011;17(11):1391-401.

15. Mallon DH, Summers DM, Bradley JA, et al. Defining delayed graft function after renal transplantation: simplest is best. Transplantation 2013;96(10):885-9.

16. Ditonno P, Impedovo SV, Palazzo S, et al. Effects of ischemia-reperfusion injury in kidney transplantation: risk factors and early and long-term outcomes in a single center. Transplant Proc 2013;45(7):2641-4.

17. Zagdoun E, Ficheux M, Lobbedez T, et al. Complicated lymphoceles after kidney transplantation. Transplant Proc 2010;42(10):4322-5.

18. Shoskes DA, Hanbury D, Cranston D, et al. Urological complications in 1,000 consecutive renal transplant recipients. J Urol 1995;153(1):18-21. 
19. Schroppel B, Legendre C. Delayed kidney graft function: from mechanism to translation. Kidney Int 2014;86(2):251-8.

20. Hall IE, Reese PP, Doshi MD, et al. Delayed Graft Function Phenotypes and 12Month Kidney Transplant Outcomes. Transplantation 2017;101(8):1913-23.

21. Berli JU, Montgomery JR, Segev DL, et al. Surgical management of early and late ureteral complications after renal transplantation: techniques and outcomes. Clin Transplant 2015;29(1):26-33.

22. Di Carlo HN, Darras FS. Urologic considerations and complications in kidney transplant recipients. Adv Chronic Kidney Dis 2015;22(4):306-11.

23. Haferkamp A, Dorsam J, Mohring K, et al. Ureteral complications in renal transplantation with more than one donor ureter. Nephrol Dial Transplant 1999;14(6):1521-4.

24. Ghazanfar A, Zaki MR, Pararajasingam R, et al. Outcome of kidney transplant with double ureter: a multicenter study. Exp Clin Transplant 2015;13(2):152-6. 
Figures and Tables

\begin{tabular}{|c|c|c|c|}
\hline Variable & $\begin{array}{l}\text { Cases } \\
n=31\end{array}$ & $\begin{array}{c}\text { Controls } \\
n=62\end{array}$ & $\mathbf{p}$ \\
\hline \multicolumn{4}{|l|}{ Receptor characteristics } \\
\hline Female gender, n (\%) & $17(54.8)$ & $34(54.8)$ & 1.0 \\
\hline Age, mean (SD) & $38.3(13.5)$ & $37.9(13.3)$ & 0.9 \\
\hline \multicolumn{4}{|l|}{ CKD etiology, n (\%) } \\
\hline Idiopathic & $13(41.9)$ & 22 (35.5) & 0.54 \\
\hline Diabetes mellitus & $5(16.1)$ & $15(24.2)$ & 0.37 \\
\hline Hypertensive & $2(6.5)$ & $0(0)$ & 0.1 \\
\hline ADPKD & $3(9.7)$ & $6(9.7)$ & 1.0 \\
\hline SLE & $4(12.9)$ & $6(9.7)$ & 0.72 \\
\hline Other & $4(12.9)$ & $13(21.0)$ & .343 \\
\hline BMI, mean (SD) & $24.2(3.8)$ & $23.8(3.8)$ & 0.89 \\
\hline Tobacco use, n (\%) & $2(6.5)$ & $7(11.3)$ & 0.71 \\
\hline$\geq 1$ HLA match, n (\%) & $8(25.8)$ & $22(35.5)$ & 0.34 \\
\hline Class 1 PARA, mean (SD) & $6.4(16)$ & $4.9(12.5)$ & 0.42 \\
\hline Class 2 PARA, mean (SD) & $4.3(11.8)$ & 5.7 (15.9) & 0.55 \\
\hline Donor-specific HLA antibodies, n (\%) & $5(13.6)$ & $11(17.7)$ & 0.84 \\
\hline Pretransplant diuresis, $\mathrm{n}(\%)$ & $20(64.5)$ & $46(74.2)$ & 0.332 \\
\hline Dialysis to transplant time, months, mean (SD) & $36.7(38.4)$ & $28.8(28)$ & 0.9 \\
\hline \multicolumn{4}{|l|}{ Donor characteristics } \\
\hline Female gender, $\mathrm{n}(\%)$ & $15(48.4)$ & $35(56.5)$ & 0.46 \\
\hline Age, mean (SD) & $40.2(12.3)$ & $38.7(13.8)$ & 0.58 \\
\hline \multicolumn{4}{|l|}{ Donor type, n (\%) } \\
\hline Living & $15(48.4)$ & $30(48.4)$ & 1.0 \\
\hline Deceased & $16(51.6)$ & $32(51.6)$ & \\
\hline Related donor, n (\%) & $12(38.7)$ & $23(37.1)$ & 0.88 \\
\hline Expanded criteria donor, n (\%) & $6(19.4)$ & $4(6.5)$ & 0.58 \\
\hline \multicolumn{4}{|l|}{ Comorbidities, n (\%) } \\
\hline Diabetes mellitus & $3(9.7)$ & $3(4.8)$ & 0.397 \\
\hline Dyslipidemia & $3(9.7)$ & $4(6.5)$ & 0.682 \\
\hline Hypertension & $4(12.9)$ & $2(3.2)$ & 0.09 \\
\hline GFR by MDRD, mL/min/1.73m², mean (SD) & $87(45)$ & $98.5(45.5)$ & 0.57 \\
\hline
\end{tabular}

ADPKD: autosomal dominant polycystic kidney disease; BMI: body mass index; CKD: chronic kidney disease; GFR glomerular filtration rate; HLA: human leukocyte antigen; SD: standard deviation; SLE: systemic lupus erythematosus. 


\begin{tabular}{|l|c|c|c|}
\hline Table 2. Kidney transplant perioperative features for cases and controls \\
\hline Variable & $\begin{array}{c}\text { Cases } \\
\mathbf{n = 3 1}\end{array}$ & $\begin{array}{c}\text { Controls } \\
\mathbf{n = 2}\end{array}$ & $\mathbf{p}$ \\
\hline Perioperative features & $670.3(636.3)$ & $676(611)$ & 0.78 \\
\hline Ischemia time, minutes, mean (SD) & $306.9(91.7)$ & $276.4(71)$ & 0.72 \\
\hline Surgical time, minutes, mean (SD) & $334.6(202.1)$ & $323.5(212)$ & 0.98 \\
\hline Surgical bleeding, mL, mean (SD) & $6(19.4)$ & $16(25.8)$ & 0.47 \\
\hline$\geq 2$ graft arteries, n (\%) & $1(3.2)$ & $3(4.8)$ & 1.0 \\
\hline$\geq 2$ graft veins, n (\%) & $\mathbf{4 ( 1 2 . 9 )}$ & $\mathbf{0}$ & $\mathbf{0 . 0 1}$ \\
\hline Ureteral duplication, n (\%) & $26(83.9)$ & $49(79)$ & 0.57 \\
\hline Extravesical implant, n (\%) & $\mathbf{7 ( 2 2 . 6 )}$ & $\mathbf{5 ( 8 . 1 )}$ & $\mathbf{0 . 0 4 9}$ \\
\hline Delayed graft function, n (\%) & $1(3.2)$ & 0 & \\
\hline Perioperative complications, n (\%) & $2(6.5)$ & $4(6.5)$ & 0.15 \\
Lymphocele & $\mathbf{3 ( 9 . 7 )}$ & $\mathbf{0}$ & $\mathbf{0 . 0 3}$ \\
Hematoma & $2(6.5)$ & 0 & 0.1 \\
Urinoma & 0 & $1(1.6)$ & 1.0 \\
Urinary fistula & & & \\
Arterial anastomosis stenosis &
\end{tabular}

SD: standard deviation.

\begin{tabular}{|c|c|c|c|}
\hline Variable & $\begin{array}{l}\text { Cases } \\
\mathbf{n}=31\end{array}$ & $\begin{array}{c}\text { Controls } \\
n=62\end{array}$ & $\mathbf{p}$ \\
\hline \multicolumn{4}{|l|}{ Post-transplant followup } \\
\hline Basal post-transplant creatinine, mean (SD) & $1.2(0.35)$ & $1.18(0.31)$ & 0.39 \\
\hline $\begin{array}{l}\text { Post-transplant basal GFR by MDMR, } \\
\mathrm{ml} / \mathrm{min} / 1.73 \mathrm{~m}^{2} \text {,mean (SD) }\end{array}$ & $71(32.2)$ & $71(20.4)$ & 1.0 \\
\hline 5-day graft ultrasound anomalies & & & \\
\hline Hydronephrosis, n (\%) & $4(12.9)$ & $3(4.8)$ & 0.27 \\
\hline Perigraft fluid collection, n (\%) & $2(6.5)$ & $7(11.3)$ & 0.7 \\
\hline Distal intrarenal resistive index, mean (SD) & $0.64(0.12)$ & $0.65(0.08)$ & 0.07 \\
\hline CMV infection, n (\%) & $2(6.5)$ & $4(6.5)$ & 1.0 \\
\hline BK virus infection & 0 & 0 & - \\
\hline Positive post-surgery urine culture & $29(29)$ & $23(37.1 \%)$ & 0.44 \\
\hline
\end{tabular}

CMV: cytomegalovirus; GFR: glomerular filtration rate; SD: standard deviation. 
Table 4. Factors associated with an increased risk of ureteral stenosis development

\begin{tabular}{|l|c|}
\hline Variable & Odds ratio (95\% CI) \\
\hline Delayed graft function & $3.3(0.96-11.52)$ \\
\hline Urinoma & $\mathbf{3 . 2 ( 2 . 3 6 - 4 . 3 7 )}$ \\
\hline Ureteral duplication & $\mathbf{3 . 2 9} \mathbf{( 2 . 4 0 - 4 . 5 1 )}$ \\
\hline
\end{tabular}

CI: confidence interval 